\title{
The Physician as a Locus of Authority, Responsibility, and Operational Control of Medical Systems
}

\author{
Bruce A. Friedman, M.D., and James B. Martin, Ph.D.
}

\begin{abstract}
Physicians are commonly being excluded from meaningful participation in the planning, implementation, and operation of automated medical systems in hospitals. The authors advocate a rapid shift toward greater physician involvement in such systems, arguing that such a shift is desirable, feasible, and also inevitable. After reviewing the organization of information systems in hospitals, the authors describe the manner in which physician control of medical systems adds to the worth of such systems by enhancing the quality and efficiency of health care delivery. The proposed information system management role of physicians is characterized in terms of authority, responsibility, and operational control. Finally, advice is offered from an organizational perspective for establishing a physician as the hospital Medical Information Director.
\end{abstract}

\section{INTRODUCTION}

The delivery of health care is rapidly becoming both more challenging and frustrating for physicians who are required to analyze data from multiple and increasingly complex data bases and then make both diagnostic and therapeutic decisions for their patients on the basis of these data. The word "analyze" is used here to mean the assimilation and manipulation of data, and their subsequent conversion into knowledge to buttress clinical decisions. The complexity of the physicians' decision-making task is compounded by the necessity for them to restrain health care costs while maintaining high standards for the quality of care.

The increasing frustration of physicians derives from many sources. A significant contribution is certainly a paucity of sophisticated information management tools in hospitals and individual practice settings. Physicians urgently need greater computer assistance in order to access and manipulate medical data and thus to enhance the quality and efficiency of the health care services they provide to patients.

From the Departments of Pathology and Health Services Management and Policy, The University of Michigan Medical School and School of Public Health, Ann Arbor, Michigan 48109. 
Although the need for improved information management tools is becoming better recognized, physicians are more often than not being excluded from meaningful participation in the planning, implementation, and operation of automated medical systems in hospitals. ${ }^{1}$ This exclusion occurs, in part, because such activities usually take place within the administrative domain of hospitals and also because physicians' heretofore tangential participation in hospital computer affairs may have created the impression among some hospital administrators that physicians are indifferent towards the topic.

Regardless of the underlying cause, this lack of direct physician involvement in hospital information system affairs will have serious long-term consequences. Decisions are now being made regarding the planing and implementation of medical systems in hospitals that will have a profound impact on the structure and practice of medical care for years to come. Further, it is probable that these decisions will be seriously flawed because of the lack of sufficient physician input.

The basic thrust of this article is that a rapid shift toward greater physician involvement in the "control" of hospital medical systems is both desirable and feasible. Because information systems are almost universally recognized as sources of power within organizations - hospitals being no exception-such a shift will obviously not occur without compelling evidence that important benefits will be derived from it. We therefore frame our discussion about the need for such a shift in system control in terms of the manner in which the quality and efficiency of medical systems will be enhanced by it.

In order to discuss the issue of physician control of medical systems, we first classify the total range of hospital systems, delineating the subset of medical systems in the process. After describing the manner in which physician involvement enhances the value of medical system output, we define the proposed management role of systems by physicians in terms of the concepts of authority, responsibility, and operational control. In order to operationalize our proposed approach, we close by offering practical advice from a hospital organizational perspective for establishing physicians as the primary locus for these three components of system control.

\section{Defining Information Systems in Hospitals}

The ensuing discussion of the role of physicians in enhancing the quality and efficiency of medical systems is built around a classification structure for all hospital information systems. It is, therefore, necessary to provide at the outset some details about this structure - the major components are core, business/financial, communications/networking, medical support, medical documentation, and departmental management. ${ }^{1}$

The first three components can be described as primarily administrative whereas the latter three are primarily medical. The major advantage of such a dichotomous view of hospital systems is that it clearly differentiates the predominantly medical components of hospital information systems from the more administrative ones, and thus highlights the potential role of physicians regarding control of the medical applications.

Core Systems. Core systems consist of the basic centralized or common hospital functions, including patient registration, admission-discharge-transfer (ADT), and appointments/scheduling. The word common in this context refers to any function that is utilized throughout a hospital rather than to the frequency of its use. Those functions which cut broadly across organizational boundaries should, with few exceptions, be 
earmarked for performance in a centralized, rather than distributed, fashion. Such an approach avoids system redundancy, reducing both information processing costs and errors caused by multiple entry of identical data into separate data bases.

Although order entry/result reporting (OE/RR) is clearly a core system from the perspective of a common hospital function, it may not necessarily fit best within the administrative domain. Historically, $\mathrm{OE} / \mathrm{RR}$ has been viewed as an administrative core application because it is the primary mechanism for capturing charges for services rendered in hospitals. The major raison d' etre for $\mathrm{OE} / \mathrm{RR}$, however, is electronic ordering of ancillary medical services (e.g., laboratory tests, radiologic procedures, or drugs from the pharmacy) with subsequent reporting of test or procedure results.

The message switching function of $\mathrm{OE} / \mathrm{RR}$, it could be reasonably argued, belongs in the medical domain because of the preponderance of medical transactions handled. From this perspective, a "front-end server," implemented to handle OE/RR tasks for the full spectrum of distributed clinical computers in a hospital, belongs in the medical domain. A server is best thought of as a computer which acts as an intermediary between the user and other computers.

Business/Financial and Communications/Networking Systems. Business/financial systems consist of traditional business-oriented functions such as accounts receivable, accounts payable, general ledger, and payroll. Extending the logic of our previous comments about whether OE/RR is an administrative or medical application, the two systems discussed in this section can be viewed as the only "purely" administrative applications in our general categorization.

Because business applications were introduced into hospitals at an early date and are thus well-entrenched in the fabric of such institutions, automated medical applications have often been inappropriately "'spun-off' from these existing systems. We believe that such an approach is backward. Medical systems should be built to directly support the central mission of hospitals - the delivery of health care services. Using such an approach, administratively oriented systems should be driven by the data byproducts of the medical systems, not vice versa.

Communications/networking consists of the physical media as well as the logical structure that tie together the disparate components of hospital information systems. The physical components of the communications/networking system consist of elements such as cable and network interface units which provide connections from the cable to attached terminals and computers. The logical structure is the intelligence of the network which understands, for example, how to route messages to individuals and to computers as well as how to synchronize data elements across systems. Communications/networking is best characterized as the "electronic glue" that links together all hospital information components.

As information system mature, we believe that the communications/networking function will be viewed increasingly as a utility similar to heat and electricity. Thus, while such a capability is absolutely necessary within a hospital, we conclude that responsibility for communications/networking will ultimately not be viewed as being of critical importance in an internal hospital organizational and political sense. Electrical and telephone services are not doled out in support of strategic goals-they tend rather to be provided in a neutral and institution-wide fashion. The same should apply to communications/networking services. This statement, of course, is predicated on the assumption 
that sufficient development and operating resources are allocated to the communication system in order to allow it to function as a utility.

Medical Documentation, Medical Support, and Departmental Systems. The first of the more medically oriented components of our hospital information system structure is medical documentation. This component encompasses what is commonly understood today to be the medical record as well as other systems closely allied to the medical record such as quality assurance, utilization review, infection control, and discharge planning. The medical documentation system is critical for physicians because it generates or accesses the data upon which the vast majority of physicians' diagnostic and therapeutic decisions are made.

As the data input streams to the medical record become totally automated (e.g., laboratory results; digitized x-ray images; narrative radiology reports, physician and nursing progress notes), the medical record will evolve gradually into a completely electronic data base. When this evolution is complete, the medical documentation system will become comparable to other computer nodes on the hospital network such as the laboratory or radiology information systems. Furthermore, the large numbers of personnel currently handling hardcopy medical record documents will be replaced by a cadre of personnel who understand both medical documentation and computer technology.

Making this same point from a different perspective, the automated medical record system will come to be perceived as a medical documentation tool and not primarily as a system intended to support billing or as a means for resource monitoring. ${ }^{2}$ A misunderstanding of the proper function of the medical record, coupled with ambiguity about system design goals, has resulted in inefficient automated medical systems in many hospitals which hinder rather than support clinical activities.

Medical support systems are generally synonymous with "expert systems"' and use artificial intelligence (AI) techniques to emulate the decision-making behavior of an expert in a specialized knowledge-intensive field. ${ }^{3}$ The HELP system, developed by Warner and associates, is an example of a holistic hospital information system (HIS) that was designed to aid in patient care and which integrates both information and knowledge processing. ${ }^{4-6}$ ONCOCIN, an example of an advanced expert system designed for use after a diagnosis has been reached by a physician, focuses on the chemotherapy management of patients with cancer. ${ }^{7}$ It is very important to note that the medical documentation data base will serve as the primary reference data base for medical support systems such as these.

Departmental management systems directly support the mission of individual departments such as Pathology and Radiology. Such systems were among the first medical applications to be implemented in hospitals and continue to be among the most successful. It is important to restate that such departmental systems must be interfaced with the medical documentation system and are essential components of the electronic medical record.

Departmental systems are a microcosm of a comprehensive HIS in that they combine both administrative and medical features. Components of departmental systems may therefore directly overlap various applications described above as core systems. For example, vendor-supplied Laboratory Information Systems (LISs) usually have their own $\mathrm{OE} / \mathrm{RR}$ and charge generation modules. In a properly designed institutional system, these redundant features of departmental systems are performed by a core server, conserving 
departmental computer system overhead as well as providing a "seamless interface" to the user.

Within our classification structure, nursing services are best considered as a departmental system because of the great similarity between the applications performed by a Nursing Information System (NIS) and those performed by an LIS or a Radiology Information System (RIS). Within the domain of both nursing and clinical laboratory services, discrete functions are performed which are unique to that domain and which do not generate data of sufficient general interest to be included in the medical documentation data base. Quality assurance activities within nursing and the various clinical laboratories come readily to mind as examples of such local functions. On the other hand, both the nursing service and the clinical laboratories generate output information (e.g., clinical nursing observations about patients or actual laboratory results) of sufficient interest and relevance to be included in the medical documentation data base.

Dowling has commented on the major factors that have inhibited the development of NISs in the past; his list illustrates both conflicts within the nursing profession as well as previously documented pitfalls in computer system development. ${ }^{8}$ The factors are: (a) a lack of agreement within the nursing profession as to its role and decision-making prerogatives; (b) a lack of meaningful ideation in NIS design, resulting in a bias toward current operations and problems, as opposed to future applications; and (c) an inappropriate willingness on the part of nurses to defer to "computer professionals" for NIS design decisions.

\section{Physicians and Value-added Processes}

The manner in which physician involvement with medical systems adds to the worth of the output of such systems is related to the concept of "value-added processes." According to Taylor, "an information system . . . is a series of value-adding processes (emphasis by author), the results of which help the users . . to make choices or which assist them in clarifying problems." 9 In other words, the merit of a system lies in its success in facilitating better decisions and solutions. Taylor describes criteria to assess the extent to which systems are useful; that is, add value. These criteria include quality, noise reduction, ease of use, adaptability, time-saving, and dollar cost-saving. Noise reduction is a jargon term for the process of including, excluding, or otherwise selecting relevant data. ${ }^{10}$

According to Taylor, in order to define and delineate the value-added processes of any information system, it is essential to understand the data flow in the environment served by the system, what the system is intended to do and for whom, and what effect the system will have on those who use it. We believe that physicians are uniquely qualified by their training, hospital role, and working knowledge of medical care to design and implement the value-added processes provided by hospital medical systems.

Upon reflection, it becomes clear that the values added by medical systems are, in essence, enhancements to the quality and efficiency of health care delivery. The quality dimensions of medical care which necessitate the participation of physicians in medical systems are: (a) the accuracy and integrity of the medical data base; (b) the increasingly important role of automated medical systems for assessing and monitoring the medical care process; and (c) the medico-legal imperative which requires that physicians under- 
stand the medical data base and the algorithms used to manipulate these data. The efficiency dimensions of automated systems such as a rapid turnaround time for laboratory results or the ability to filter out nonessential data for the user are self-evident and we will not belabor them here.

The critical question, then, regarding the control of medical systems in hospitals is not whether administrators are willing to take the risk of creating an environment congenial to physician involvement with such systems. Rather, the question reduces to whether administrators can afford the risk of not pursuing such a course of action. We believe that the obvious answer is that they cannot.

\section{Defining Locus of Authority, Responsibility, and Operational Control of Medical Systems}

We strongly believe that physicians should serve as the primary locus of authority, responsibility, and operational control for medical systems. These three terms require additional clarification. We use the term authority to encompass the concept of management control ${ }^{11}$ of information systems by physicians, including active participation in both system planning as well as overall decision-making activities. Management control, and hence authority, also includes the determination of what resources are necessary and available to achieve the selected goals.

Responsibility is used to mean that physicians should be held directly accountable for the functions and functioning of the medical systems. Using the vernacular, responsibility means that "the buck stops here." Operational control of medical systems, in contrast to management control, means that physicians should direct the daily real-time allocation of available resources and operations in order to achieve the goals established via management control. We believe that it is unworkable for individuals to bear authority and responsibility for information systems without simultaneously exercising operational control. Physicians cannot assume de jure control of those information systems which directly affect patient care decisions while day-to-day de facto control is actually exercised by administrators or computer personnel. ${ }^{12}$

Physicians currently have responsibility for the delivery of patient care in hospitals, but exercise neither authority nor operational control for the medical documentation system which is critical for their ongoing medical decision-making processes. This situation will become increasingly untenable as increasingly larger portions of the medical documentation system become automated. Unless substantial changes occur, physicians will increasingly lose control of their own decision-making tools. In support of this view, Brannigan and Dayhoff provide a detailed and cogent analysis of the extent to which the introduction of computers in hospitals has served to challenge the role of physicians as medical "decisionmakers" and created conflicts over the control of hospital information. ${ }^{13}$

Our goal in this section has been to refine and expand the definition of control of systems away from a highly politicized view and toward the notion of authority, responsibility, and operational control of systems. This was done in order to more accurately represent the future comprehensive role we envision for physicians. Keen has previously commented on the link between information and power and politics and highlighted the manner in which information systems "alter relationships, patterns of communication, and perceived influence, authority, and control." 14 
The foregoing discussion should not be interpreted to mean that we reject the idea of matrix management in hospitals. It is obvious that no group can operate autonomously in a well-run hospital today. Responsibility for medical systems must be broadly shared, but we believe that physicians must take the direct lead and initiative for controlling the process and product of medical systems.

\section{Hospital Organizational Framework to Accommodate Physician Involvement in Computer Affairs}

We clearly believe that the primary locus of authority, responsibility, and operational control for the three medically related components of hospital information systems (Medical Documentation, Medical Support, and Department Management) should lie with physicians. The manner in which this goal is actually accomplished within individual hospitals is highly dependent on the hospital size and local organizational framework for medical affairs.

Most certainly, larger hospitals should appoint a physician as Medical Information Director and this individual should operate in the upper levels of hospital management. The scope of this position should include responsibility for the Medical Records Department and central coordination of the activities of the various medically-oriented departmental information systems. Such coordination could occur through the activities of a hospital Medical Computing Group which the Medical Information Director would head. ${ }^{1}$ To link this idea to the previous discussion of authority and responsibility for systems, the Medical Information Director must also be charged with making resource allocation decisions regarding medical systems.

The key element of this plan is the need for a full-time physician with continuing responsibility for the planning, implementation, and operation of medical systems. Only such an individual working full-time would be willing and able to devote the time and concentration that such tasks demand. Korpman notes that some hospitals have attempted to fill a position such as this with an administrator, but that " an analysis of the patient care and quality component of this position leads one to conclude that in most cases only a physician has the requisite clinical skills to execute this task effectively." 2 He refers to such an individual as a "medical information specialist."

This discussion of the organizational framework for physician involvement in computer affairs is not meant to minimize the need for the development of computer expertise within the entire body of hospital physicians. Existing hospital committees, such as those addressing quality assurance and utilization review, should allocate more time toward the discussion of medical systems. In addition, there should be an overall goal of promotion of "nodes of computing excellence" in the hospital in addition to foci within the specialized departmental system groups.

\section{CONCLUSION}

There remain a number of major barriers to achieving greater physician responsibility and control of automated medical systems: resistance on the part of hospital administrators who currently control administrative systems and are well positioned to control the evolv- 
ing medical systems; the lack of a sufficiently large cadre of physicians trained in medical informatics; an attitude on the part of some physicians that many information processing activities are "housekeeping" details and not relevant for them as highly-trained professionals; anxiety on the part of physicians about the need to learn new techniques and display their ignorance in the process; and a relative lack of useful and commercially available software to support sophisticated medical applications.

These problems notwithstanding, the future direction is clear. Physicians must forge ahead and bring their multifaceted skills to bear on the task of integrating automated systems into the medical care process. Failure to do so will only cause a decline in both the quality and efficiency of health care, but also in the professional status of physicians as well.

\section{REFERENCES}

1. Friedman, B.A., and Martin, J.B., Hospital information systems. The physician's role. JAMA 257:1792, 1987.

2. Korpman, R.A., Using the computer to optimize human performance in health care delivery. Arch. Pathol. Lab. Med. 111:637-645, 1987.

3. Duda, R.O., and Shortliffe, E.H., Expert systems research. Science 220:261-268, 1983.

4. Pryor, T.A., Gardner, R.M., Clayton, P.D., and Warner, H.R., The HELP system. J. Med. Syst. 7:87$102,1983$.

5. Warner, H.R., Computer-Assisted Decision Making, Academic Press, New York, 1979.

6. Blum, B.I., Clinical information systems-A review. In Medical Informatics [Special Issue]. West. J. Med. 145:791-797, 1986.

7. Shortliffe, E.H., Medical expert systems-Knowledge tools for physicians. In Medical Informatics [Special Issue]. West. J. Med. 145:830-839, 1986.

8. Dowling, A.F., Nursing functions and computer support. J. Med. Sys. 9:91-96, 1985.

9. Taylor, R.S., Value-Added Processes in Information Systems, Ablex Publishing Corporation, Norwood, N.J., 1986, p. 5.

10. Taylor, R.S., Value-Added Processes in Information Systems, Ablex Publishing Corporation, Norwood, N.J., 1986, p. 50.

11. Anthony, R.N., and Dearden, J., Management Control Systems, Richard D. Irwin, Inc., Homewood, Ill., 1976.

12. Friedman, B.A., Pathologists, computers, and control of the clinical laboratory database. Pathologist 40:40-46, 1986.

13. Brannigan, V.M., and Dayhoff, R.E., Medical informatics. The revolution in law, technology, and medicine. J. Leg. Med. 7:1-53, 1986.

14. Keen, P.G.W., Information systems and organizational change. Communications ACM 24:24-33, 1981. 